\title{
Analysis of crystallization kinetics and shape memory performance of PEG-PCL/MWCNT based PU nanocomposite for tissue engineering applications
}

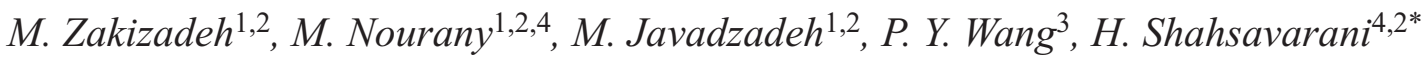 \\ ${ }^{1}$ AmirKabir University of Technology, Department of Polymer Engineering and Color Technology, Tehran, Iran \\ ${ }^{2}$ Laboratory of Regenerative Medicine and Biomedical Innovations, Pasteur Institute of Iran, Tehran, Iran \\ ${ }^{3}$ Shenzhen Key Laboratory of Biomimetic Materials and Cellular Immunomodulation, Shenzhen Institute of Advanced \\ Technology, Chinese Academy of Sciences, Shenzhen, 518055 Guangdong, China \\ ${ }^{4}$ Department of Cellular and Molecular Sciences, Faculty of Bioscience and Biotechnology, Shahid Beheshti University, \\ Tehran, Iran
}

\begin{abstract}
In this work, a series of reactive in-situ polyurethane (PU) nanocomposites based on the triblock copolymer of $\mathrm{PCL}_{1000}-\mathrm{PEG}_{1000}-\mathrm{PCL}_{1000}$, chemically cross-linked by hydroxyl-functionalized MWCNTs (um-MWCNT) and PCL-grafted MWCNTs (mod-MWCNT), were synthesized. In order to optimize the shape memory performance, crystallization mechanisms of the soft domains were tuned. The nanoparticles, acting as phase controller of the block copolymer, affected the chain's confinement and crystals' morphology leading to a wide range of shape fixity (84-100\%) and shape recovery (78$97 \%$ ) ratios. Non-isothermal crystallization studies revealed that using mod- MWCNTs increased the melting temperature $\left(T_{\mathrm{m}}\right)$ as an indication of higher thermal stability of the formed crystallites. Moreover, isothermal DSC measurements, fitted to the Avrami equation, were used to measure the changes in the growth rate and morphological features of the formed crystallites. The results indicated an increase in Avrami exponent $(n)$ from 1.43 to 3.11, and crystallization half-time $\left(t_{0.5}\right)$ decreased from 6.16 to 2.67 minutes for crystallization temperature $\left(T_{\mathrm{c}}\right)$ of $-25^{\circ} \mathrm{C}$, attributed to the effect of PCL grafts on PUs' microstructure. In addition, the results of cell viability, evaluated by HFF cells, proved a proper cytocompatibility. Culturing hMSCs also showed good adhesion and cell spreading, as a function of hydrophilicity. The optimum sample, containing $0.5 \%$ PCL-g-MWCNT, showed $97 \%$ shape recovery at body temperature $\left(37^{\circ} \mathrm{C}\right)$.
\end{abstract}

Keywords: thermal properties, in-situ PU nanocomposite, shape memory performance, crystallite morphology transformation, hMSCs culturing

\section{Introduction}

Shape memory polymers (SMPs) are smart and responsive materials with the ability to store applied external stress and recover the original shape [1]. They are used in various applications such as actuators, sensors, and tissue engineering [2]. Polyurethanes (PUs) are known for their high thermal-induced shape recovery behavior with hard domains acting as permanent component (PC) and soft domains as switching components (SC). PC, with a network structure, memorizes the permanent shape of the specimen through prohibiting irreversible sliding of polymer chains. The reason for the excellent shape fixity and recovery of polyurethane samples is the ability of the soft domain chains to fix the temporary shape by selecting glass transition $\left(T_{\mathrm{g}}\right)$ or melting temperature $\left(T_{\mathrm{m}}\right)$ as switching point, and the ability of hard domains (physical networks) to regain the

${ }^{*}$ Corresponding author, e-mail: hosein.shahsavarani@gmail.com (C) BME-PT 
original shape $[3,4]$. Control over the structure of SC and PC plays a key role in the synthesis of a thermally-induced SMP with ideal shape fixation (SF) and recovery (SR) [5, 6]. Moreover, positive physical interaction between polymer chains and high elastic modulus nanoparticles can increase the elasticity of the system with an improving effect on their shape memory behavior [7]. Utilizing high elastic nanoparticles with different morphologies in thermoplastic polyurethanes (TPUs) would have different effects on the elasticity of the nanocomposites and, consequently, on shape memory parameters [8-10].

Controlling the microstructure of polymers is a promising way to reach optimal shape-memory performance. For a polymeric system of two incompatible polymers, there are many ways to reduce the scale of phase separation, including blending, copolymerization, and using compatibilizer. Block copolymerization of two incompatible polymers is an efficient way of transforming macrophase separation into microphase separation [11]. The extent of microphase separation between two blocks of a block copolymer depends on the extent of the repulsive interaction $\left(\chi_{\mathrm{AB}}\right)$ and block chain length $(N)$ [12]. For a system of two semi-crystalline polymers, the phase transformation has an enormous impact on crystallization extent $\left(X_{\mathrm{C}}\right)$ and kinetics of the blocks (reducing $T_{\mathrm{m}}$ and $T_{\mathrm{c}}$ ). A change in polymer chain dynamics can also cause a change in crystallization thermodynamic and kinetics. According to the literature, the physical confinement of polymer chains reduces their dynamics (an increase in $T_{\mathrm{g}}$ ), which consequently limits the ability of the semi-crystalline polymers to form stable crystallites $[13,14]$. A few reports on the crystallization behavior of the reactive in-situ PU nanocomposites reported a tremendous reduction in $T_{\mathrm{m}}, T_{\mathrm{c}}$, and $X_{\mathrm{C}}$ with an increase in $T_{\mathrm{g}}[14,16]$.

Poly( $\varepsilon$-caprolactone) (PCL), as the most commonly used semi-crystalline polyester in tissue engineering applications, possess a hydrophobic and biodegradable nature, with a relatively low biodegradation rate $[17,18]$. Low molecular weight PCL with terminal hydroxyl groups is used as polyol for the synthesis of an ester-based polyurethane (PU) with a lowered crystallization extent, resulting in improved hydrophilicity and biodegradation rate [19]. Synthesis of PCL proceeds through ring-opening polymerization (ROP) of $\varepsilon$-caprolactone in the presence of $\mathrm{Sn}(\mathrm{Oct})_{2}$ as a catalyst in the temperature range of $95-120^{\circ} \mathrm{C}$, and the nature of the reaction is pseudo-anionic, which limits the polydispersity of the polymer [2022]. Polyethylene glycol (PEG) is highly hydrophilic and biocompatible with a high crystalline extent used for the synthesis of biocompatible hydrogels for drug delivery and tissue engineering applications [23]. PEG and PCL blends show repulsive interaction, resulting in a macrophase-separated blend at equilibrium $[24,25]$. The polyols in the PU structure show a decrease in crystallization extent and temperature compared to pure polyols due to the high interfacial tension between soft and hard domains and the presence of a covalent bond between them [26]. Due to the reduction in the crystallization extent of soft segments and a tendency for having an amorphous state at the rubbery region, X-ray analysis of most PUs show a broad bell-like peak around $2 \theta=19.6^{\circ}$, indicating the amorphous nature of the soft domain [27]. Cold crystallization is a phenomenon, which occurs in polymers with low chain dynamics, shows itself as a crystallization peak in the heating regime after glass transition temperature [28]. The reason for the occurrence of cold crystallization for soft segments in a PU structure can be due to the highly limited chain dynamics as they are connected to hard domains [29]. Phua et al. [30] reported an increment in storage modulus of the polyether-based polyurethane reinforced with dopamine-modified clay after glass transition temperature $\left(T_{\mathrm{g}}\right)$ and assigned it to the cold crystallization phenomenon. Liu et al. [16] reported the synthesis of PEG/PCL-based polyurethane hydrogels using cellulose nanocrystals (CNCs) as a crosslinking agent and MDI as diisocyanate. They reported a reduction in crystallization extent and temperature $\left(T_{\mathrm{c}}\right)$ down to $42^{\circ} \mathrm{C}$ but did not report any special change in possible phase separation between PCL and PEG homopolymers. Pei et al. [15] reported the synthesis of an in-situ polyether-based polyurethane elastomer nanocomposite of PTMG and a low amount of CNC. The damping factor $(\tan \delta)$ of the pure polyurethane shows an increase in the temperature range of 50 to $90^{\circ} \mathrm{C}$, while the nanocomposites did not show any increase in this temperature range, as an indication of a change in their long-range mobility. The storage modulus of the polyurethane nanocomposites showed a slight increase in the glassy region in addition to enhanced thermal stability at higher temperatures.

In this work, we studied the synthesis and characterization of reactive in-situ PU nanocomposites based on $\mathrm{PCL}_{1000}-\mathrm{PEG}_{1000}-\mathrm{PCL}_{1000}$, as the soft segment 
hydroxyl functionalized MWCNTs and PCL-grafted MWCNTs nanoparticles used for cross-linking the prepolymers. Shape memory performance of the synthesized PUs was analyzed, and the effect of crystallization of the soft segments and chemical crosslinking on shape fixity and recovery was studied in detail. Cytotoxicity of the synthesized PUs was also assessed by human foreskin fibroblast (HFF) cells. Human Mesenchymal Stem Cells (hMSCs) were cultured onto the samples to analyze cell adhesion and spreading of the cultured cell in order to evaluate their potential applications in tissue engineering for further works.

\section{Experimental}

\subsection{Materials}

Dimethylformamide (DMF), 1,4-butanediol (BDO), and Hexamethylene diisocyanate (HDI) were purchased from Merck, Germany. Polyethylene glycol (PEG, $M_{\mathrm{n}}=1000 \mathrm{~g} / \mathrm{mol}$ ) was vacuum oven-dried at $60^{\circ} \mathrm{C}$ for 72 hours prior to use. MWCNTs with an average length of $25 \mu \mathrm{m}$ and outer diameter (OD) of $15 \mathrm{~nm}$ were purchased from Neutrino co. $\varepsilon$-caprolactone monomer and $\mathrm{Sn}(\mathrm{Oct})_{2}$ were purchased from Sigma Aldrich.

\subsection{Synthesis of PCL-g-MWCNTs}

PCL grafts onto hydroxyl modified MWCNTs were performed using ring-opening polymerization (ROP) by a pseudo-anionic mechanism at $110^{\circ} \mathrm{C}$ using $\mathrm{Sn}(\mathrm{Oct})_{2}$ as a catalyst. MWCNT (1 gr) was dispersed in DMF $\left(50 \mathrm{~cm}^{3}\right)$ and probe-sonicated in four steps, every 10 minutes. Then, $20 \mathrm{~cm}^{3} \mathrm{CL}$ monomer was added to the suspension and stirred using a magnetic stirrer for $1 \mathrm{hr}$ at $110^{\circ} \mathrm{C}$. The reaction was performed under dry $\mathrm{N}_{2}$ and continuous stirring. After $24 \mathrm{hrs}$, the reaction was terminated using a few droplets of $37 \% \mathrm{HCl}$ in $5 \mathrm{~cm}^{3}$ distilled water to hydroxyl functionalize the end group.

\subsection{Synthesis of polyurethane nanocomposite}

In order to synthesize the PUs, at the first step, the $\mathrm{PCL}_{1000}-\mathrm{PEG}_{1000}-\mathrm{PCL}_{1000}$ block copolymer $(10 \mathrm{~g})$, synthesized according to the method used in our previous work [31], was added to a three-neck round bottom flask and vacuum-dried for 30 minutes at $60^{\circ} \mathrm{C}$ with a stirring rate of $200 \mathrm{rpm}$. Then, the temperature was increased to $80^{\circ} \mathrm{C}$, and HDI $(1.68 \mathrm{~g})$ was added dropwise to the flask in two hours to prepare the polyurethane prepolymers. The reaction was under a continuous purge of dry $\mathrm{N}_{2}$, and the molar ratio of HDI to the polyol was kept $3: 1$. The NCO:OH ratio in the polyurethane was kept equal to 1 for pure polyurethanes using vacuum dried 1,4-butanediol (0.6 g). MWCNTs and PCL-g-MWCNTs (PCL grafts were indicated by short red lines attached to MWCNT in Figure 1) were used to make a chemically crosslinked PU nanocomposite. Figure 1 illustrates the schematic presentation of the in-situ polyurethane reaction with two different final structures.

(PCL- $g$-)MWCNTs were dispersed in DMF and preheated at $80^{\circ} \mathrm{C}$ prior to adding it to the reaction media, and the reaction was allowed to proceed for 30 minutes. The final product was poured into a Teflon mold, and then the curing process was allowed to proceed in an oven for $12 \mathrm{hrs}$ at $80^{\circ} \mathrm{C}$. Finally, to

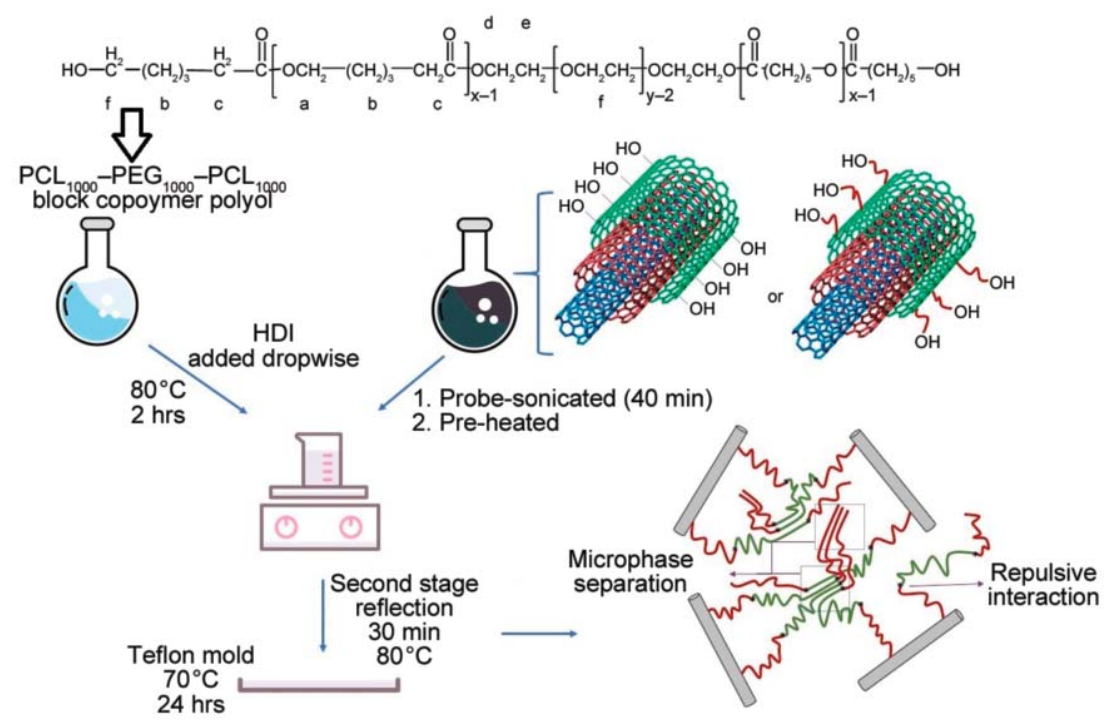

Figure 1. Schematic presentation of the synthesis procedure of the reactive in-situ PU nanocomposites and their final structure. 
ensure complete removal of DMF, the formed films were put in a vacuum oven for $12 \mathrm{hrs}$.

\subsection{Characterization techniques}

Infrared spectroscopic experiments were conducted on the prepared PU films by MOD SRG 1100G (Canada, BOMEM). NMR spectra of the block copolymer and PCL-g-MWCNT were recorded on a $500 \mathrm{MHz}{ }^{1} \mathrm{H}$ NMR (NMR INOVA $500 \mathrm{MHz}$ ) at room temperature, with CDCL3 as solvent and tetramethylsilane (TMS) as an internal reference. Differential scanning calorimetry (DSC) analysis of the samples ( $5 \mathrm{mg}$ ) was carried out under $\mathrm{N}_{2}$ atmosphere with (Mettler/Toledo) thermal analyzer at heating and cooling rates of $10{ }^{\circ} \mathrm{C} / \mathrm{min}$, and the results were recorded in $\mathrm{W} / \mathrm{g}$ unit. The PU nanocomposites were tested on a dynamical mechanical thermal analyzer (DMTA, Triton, England) working in tension mode at a frequency of $1 \mathrm{~Hz}$, strain amplitude of $0.1 \%$, a heating rate of $5{ }^{\circ} \mathrm{C} / \mathrm{min}$, and a distance between jaws of $16 \mathrm{~mm}$. The microstructure of the PUs were observed by field emission scanning electron microscopy (FESEM, TESCAN VEGAII, Czech)). All the experiments were repeated three times for each sample to analyze the repeatability of the results.

\subsection{Shape memory analysis}

The shape memory performances of the specimens were measured by DMA Triton (TA Instruments, England) using a tensile film fixture. The frequency was set at $1 \mathrm{~Hz}$. The process was conducted in four sequential steps: 1) samples were heated up to $37^{\circ} \mathrm{C}$ and kept isothermally for 5 minutes, then stretched under a force of $6 \mathrm{~N} ; 2$ ) samples were cooled to $-30^{\circ} \mathrm{C}$ to fix the temporary shape (below the crystallization temperature), and the samples were kept at this temperature for 5 minutes; 3 ) the applied tensional force was removed; 4) after 5 minutes, the samples were reheated to $37^{\circ} \mathrm{C}$ (higher than the melting temperature) at a heating rate of $10^{\circ} \mathrm{C} / \mathrm{min}$ to recover the original shape. The shape fixity ( $S F$, Equations (1)) and final shape recovery ratios ( $S R$, Equations (2)) are determined by the Equations (1) and (2):

$$
\begin{aligned}
& S F=\frac{\varepsilon_{\mathrm{u}}}{\varepsilon_{\mathrm{l}}} \cdot 100 \% \\
& S D=\frac{\varepsilon_{1}-\varepsilon_{\mathrm{r}}}{\varepsilon_{\mathrm{r}}} \cdot 100 \%
\end{aligned}
$$

where $\varepsilon_{1}, \varepsilon_{\mathrm{u}}$, and $\varepsilon_{\mathrm{r}}$ are the strain under load, the unloaded strain and the residual strain at the end of the recovery process.

\subsection{Cell viability and cell culturing procedure}

The in-vitro cytotoxicity of the PU samples was conducted using HFF cells. The punched PU samples were sterilized using ethanol (70\%) and UV radiation. The PU films were washed with PBS for three times and pre-incubated with culture media for $24 \mathrm{hrs}$. The cells were cultured into 48-well TCPS plates at the cell density of $2 \cdot 10^{4}$ cells/well with $400 \mu$ added culture media. Then, the plates were incubated at $96 \%$ humidity and $5 \% \mathrm{CO}_{2}$ atmosphere for 24 and $48 \mathrm{hrs}$ at $37^{\circ} \mathrm{C}$. The culture media was then removed. In the end, $100 \mu 1$ of MTT solution with a concentration of $0.50 \mathrm{mg} / \mathrm{ml}$ was added and incubated for $4 \mathrm{hrs}$. Then, the MTT media was removed, and $150 \mu \mathrm{l}$ DMSO was added to each well and pipetted several times. The media was then transferred to a 96-well plate, and the optical density (OD) was measured by Elisa reader at the wavelength of $570 \mathrm{~nm}$. The cell viability was measured using Equation (3):

Cell viability $[\%]=$

$=\frac{\text { Experimental optical density }(O D)}{\text { Control } O D} \cdot 100$

For seeding the human Mesenchymal Stem Cells (hMSCs) onto the PU films, at first, they were washed with distilled water in 3 steps each $8 \mathrm{hr}$, and the samples were then dried and sterilized by a UV lamp for $12 \mathrm{hrs}$. Then, the samples were placed into the 48-well TCPS plates. A suspension of the cells in DMEM (Gibco) supplemented with 10\% FBS (Gibco, UK), and $1 \%$ penicillin/streptomycin (Sigma) were transferred onto each film with a cell density of $4 \cdot 10^{4} \mathrm{cell} / \mathrm{specimen}$. The cell-seeded films were maintained for $4 \mathrm{hrs}$ at $37^{\circ} \mathrm{C}$ in an incubator with standard conditions to adhere the cells to the film. Then all samples were treated with $400 \mu 1$ of DMEM and $10 \%$ FBS. After 7 days of cultivation, the cell-cultured films were twice washed by PBS, and $2.5 \%$ glutaraldehyde was used to fix cells in $12 \mathrm{hrs}$ at $4^{\circ} \mathrm{C}$. After that, the films were washed with PBS, and in the end, the seeded cells were dehydrated in an ethanol-gradient series $(20,40,60,70,80,90,100 \%)$ for $15 \mathrm{~min}$ each. The final dehydrated cells on the films were dried via lyophilization and used for SEM imaging. 


\section{Results and discussion}

\subsection{ATR-FTIR/NMR spectroscopy}

ATR-FTIR and proton NMR spectroscopy was performed on the prepared samples to elucidate the chemical structure of the synthesized block copolymer, PCL-g-MWCNT, and the PUs, as it can be seen from Figure 2. Figure 2a shows the proton NMR of the synthesized PCL-g-MWCNT and the block copolymer. The peak in $\delta=4.7-5.3 \mathrm{ppm}$ region is characteristic of PCL, which is a polyester [32]. There is a large chemical shift for $-\mathrm{O}-\mathrm{CH}$ hydrogen from $\delta=4.7-5.3$ to $4.0-4.3 \mathrm{ppm}$, which is due to deshielding effect of the electronegative oxygen attached to the carbon nanotube and has four main peaks due to methyl group attached to it $(-\mathrm{O}-\mathrm{CH}$ $\left(\mathrm{CH}_{3}\right)^{-)}$[33]. The relative integration of the peaks available at chemical shifts of $\delta=4.13$ and $3.67 \mathrm{ppm}$ gives the molecular weight of the grafted chains being equal to $1135 \mathrm{D}$ for the chain graft. The characteristic resonance peaks of PCL $(1.35,1.54$, and $3.86 \mathrm{ppm}$ ) and PEG (3.61 and $4.19 \mathrm{ppm}$ ) are observed in the proton NMR spectrum of the synthesized block copolymer. The relative integral ratio of peaks at 3.61 and $4.19 \mathrm{ppm}$ gives the molecular weight of PEG, which is equal to $1035 \mathrm{D}$ for $\mathrm{PEG}_{1000}$. The attachment of PCL to PEG is confirmed by the presence of an overlapped shoulder at $1.47 \mathrm{ppm}$. According to Equations (4)-(6), the molecular weight of $\mathrm{PCL}_{1000}-\mathrm{PEG}_{1000}-\mathrm{PCL}_{1000}$ block copolymer was calculated using ${ }^{1} \mathrm{H}$ NMR (the used alphabets in the equations are labeled in the copolymer structure in Figure 1). Accordingly, $M_{\mathrm{n}}$ was found to be $3120 \mathrm{D}$ for the block copolymer as shown in Equations (4)-(6):

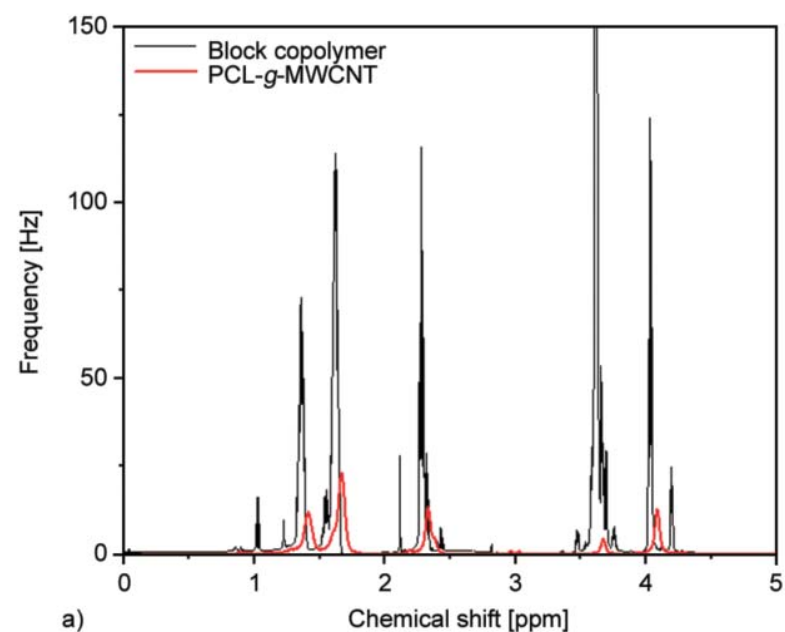

$\frac{2(2(x-1))}{I_{\mathrm{a}}}=\frac{4}{I_{\mathrm{d}}}$

$\frac{4(y-2)+4}{I_{\mathrm{f}}}=\frac{4}{I_{\mathrm{d}}}$

$\begin{aligned} M_{\mathrm{n}(\mathrm{PCL}-\mathrm{PEG}-\mathrm{PCL})} & =M_{\mathrm{n}(\mathrm{PEG})}+M_{\mathrm{n}(\mathrm{PCL})}= \\ & =44 y+2(114 x)\end{aligned}$

Figure $2 b$ presents the ATR-FTIR spectrum of the graft, block copolymer, and the synthesized PUs of um- and mod- $0.25 \%$. The absorbance peak in the range of $2800-3000 \mathrm{~cm}^{-1}$ corresponds to the stretching vibration of symmetric aliphatic groups $(\mathrm{C}-\mathrm{H})$. The absorption band at $1735 \mathrm{~cm}^{-1}$ corresponds to $\mathrm{C}=\mathrm{O}$ stretching vibrations of the carbonyl group of the ester linkage in PCL segment, indicating the presence of PCL polyester in both structures [34]. The characteristic absorption bands at 1095 and $1235 \mathrm{~cm}^{-1}$ are attributed to the $\mathrm{C}-\mathrm{O}-\mathrm{C}$ stretching vibrations of ethylene glycol $\left(-\mathrm{OCH}_{2} \mathrm{CH}_{2}-\right)$ units in PEG. The broad absorption band at $3504 \mathrm{~cm}^{-1}$ is assigned to the stretching vibrations of the hydrogen-bonded terminal and structural hydroxyl groups $(-\mathrm{OH})$ of the synthesized block copolymer and the graft [33]. According to Figure $2 b$ for the synthesized PUs, split peaks in the range of $1634-1720 \mathrm{~cm}^{-1}$ is related to different carbonyl groups available in the prepared PUs such as $-\mathrm{C}(=\mathrm{O}) \mathrm{O}-$ and $-\mathrm{NHC}(=\mathrm{O}) \mathrm{O}-$. The absorption peak available at $1543 \mathrm{~cm}^{-1}$ corresponds to $-\mathrm{NH}-$ bending deformation. Presence of a double peak for stretching vibrations of $-\mathrm{NH}$-groups in the range of $3200-3450 \mathrm{~cm}^{-1}$ represents the presence of hydrogen-bonded and free-NH-groups [35].

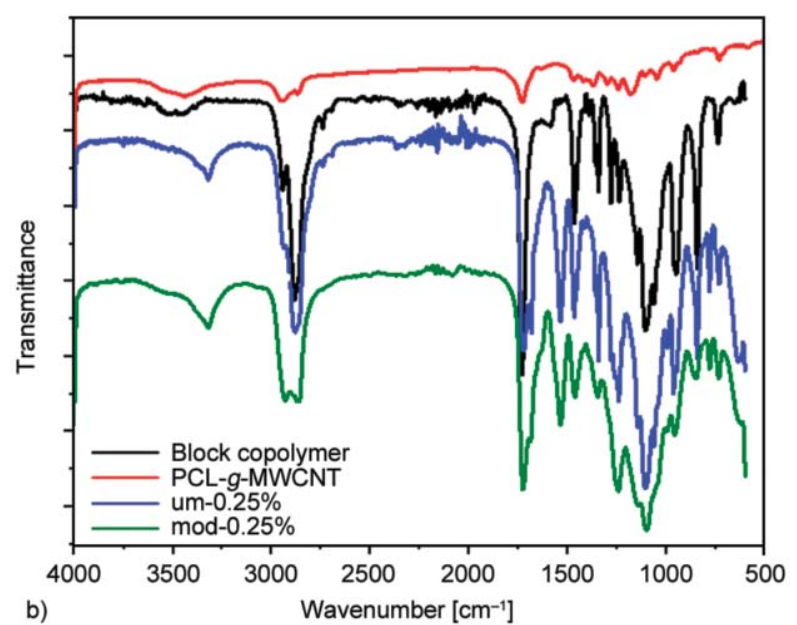

Figure 2. (a) Proton NMR spectroscopy of the synthesized block copolymer and PCL-g-MWCNT; (b) ATR-FTIR spectroscopy of the synthesized block copolymer, PCL- $g$-MWCNT, and the synthesized PUs of um- and mod- $0.25 \%$. 


\subsection{Morphological analysis}

Figure 3 illustrates FESEM images of the cryofractured PU nanocomposites of um- (Figure 3a, 3b) and mod-MWCNTs (Figure 3c, 3d). The images show a scale of $5 \mu \mathrm{m}$ to examine the morphology, dispersion, and presence of any possible clustering of the carbon nanotubes.

Based on the images, PU nanocomposites of unmodified MWCNTs showed a surface without any phase pattern with uniformly dispersed nanoparticles. However, PUs of mod-MWCNTs showed a patterned surface for all nanotube contents, which attributed to increased microphase separation.

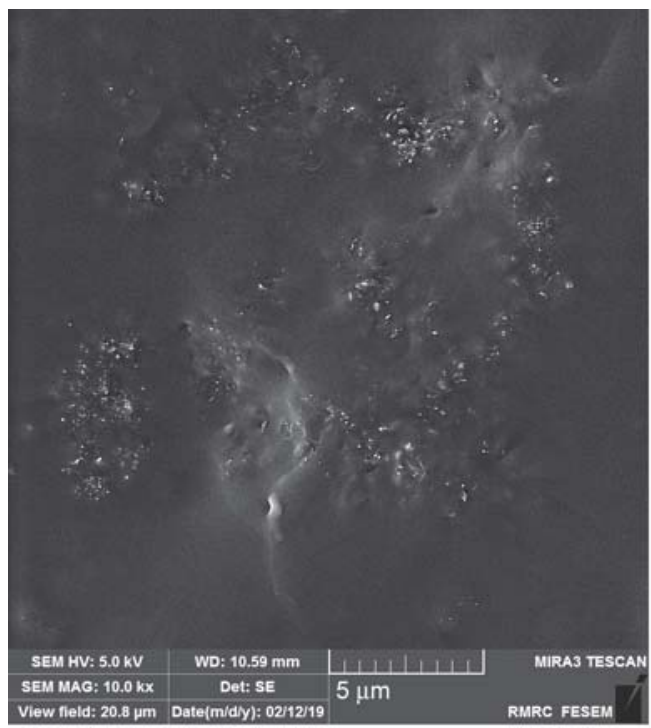

a)

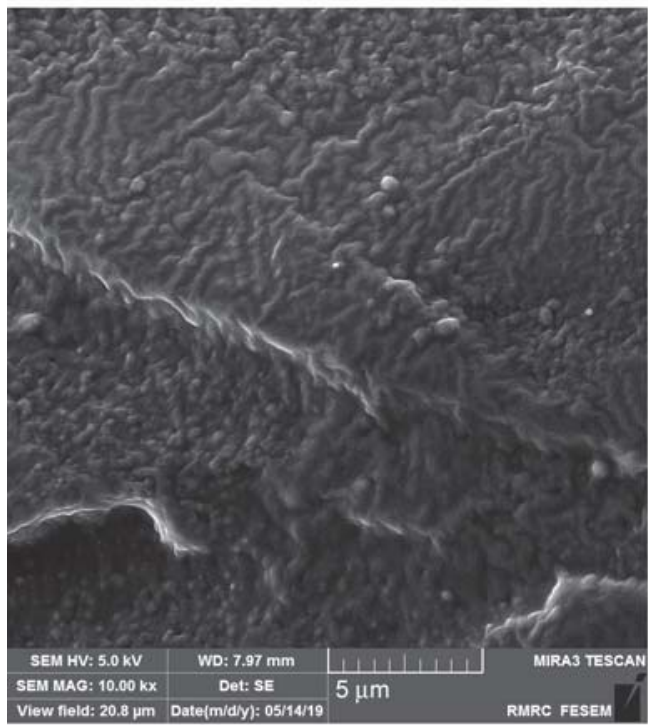

c)

\subsection{Shape memory behavior}

The shape memory behavior of the synthesized in-situ PU nanocomposites was investigated by dynamic mechanical analyzer (DMA) test, and the results are shown in Figure 4. The isothermal temperature was set to $37^{\circ} \mathrm{C}$ (body temperature and above $T_{\mathrm{m}}$ ), and the prepared specimens were stretched by applying an external dynamic force. Then, the samples were cooled down to $-30^{\circ} \mathrm{C}$ to fix the formed temporary shape. The soft segment acts as the reversible domain, and its crystalline structures tune the shape fixity $(S F)[5,36]$, and the chemical network is used to control the shape recovery $(S R)$ [37].
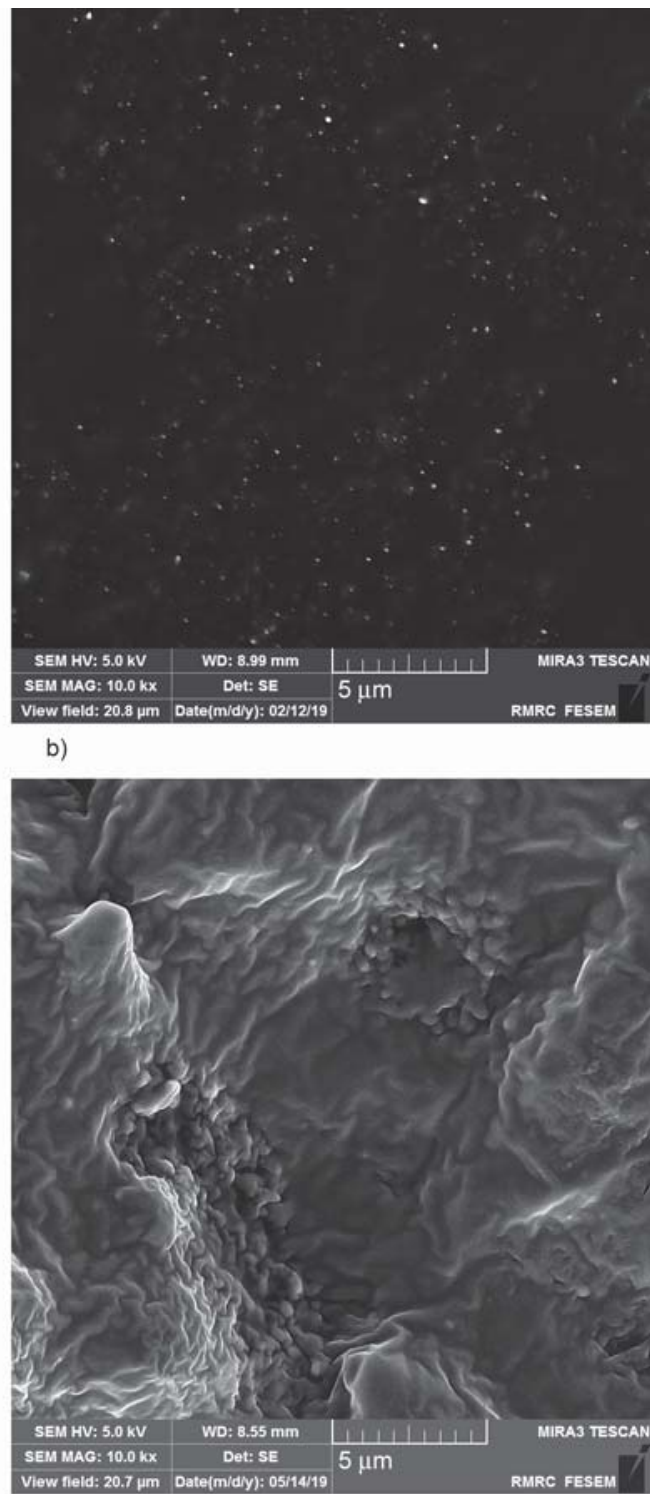

d)

Figure 3. FESEM images of PU nanocomposites of ( $a, b)$ unmodified MWCNTs, and (c, d) PCL-grafted MWCNTs for two different MWCNT contents of 0.25 , and $1 \%$ with scales of $5 \mu \mathrm{m}$, respectively. 

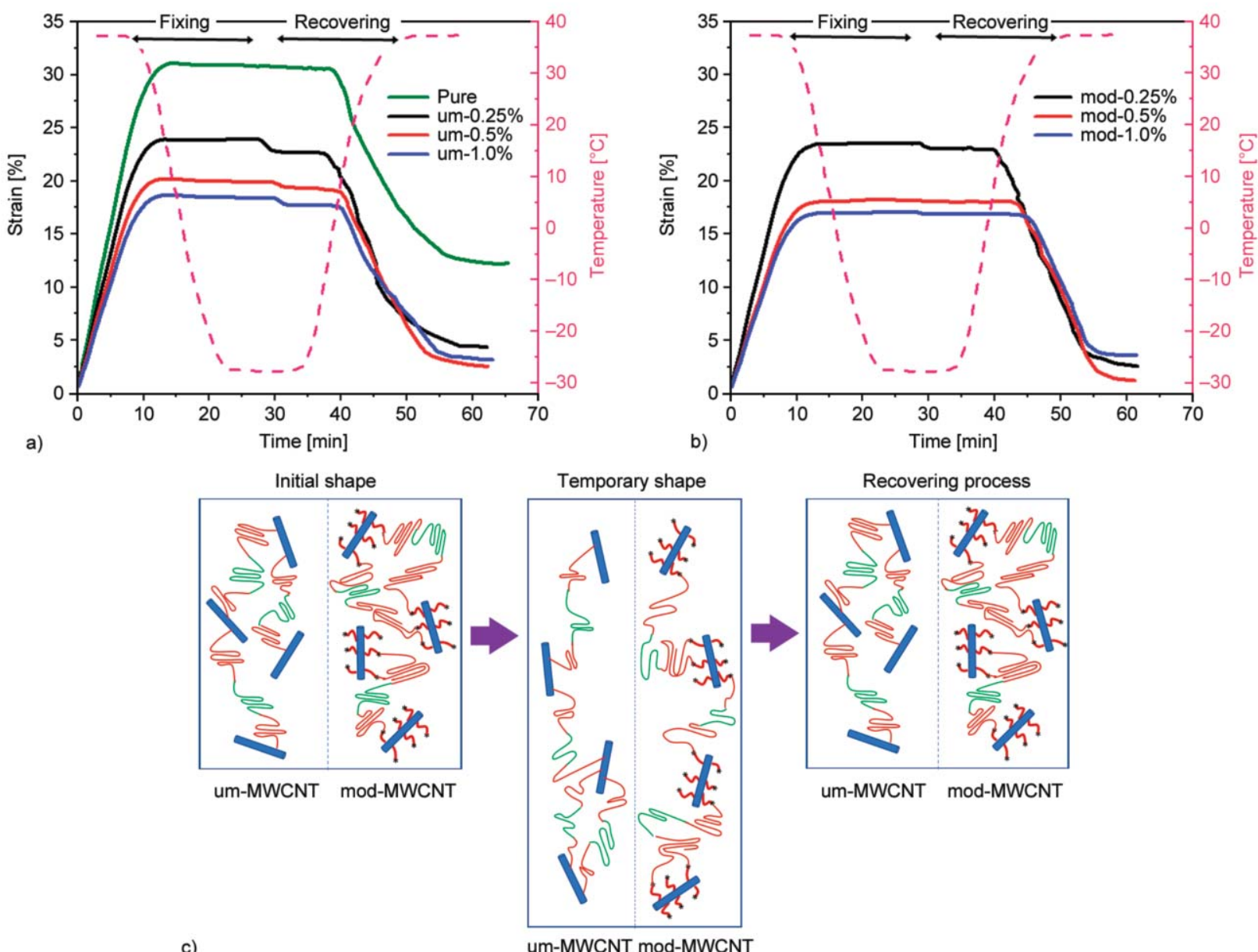

c)

um-MWCNT mod-MWCNT

Figure 4. DMA analysis of the shape memory performance for the prepared PUs of (a) pure and um-MWCNT, (b) modMWCNT, and (c) schematic representation of the shape memory process.

Chemical confinement of the tri-block copolymer between MWCNTs would decrease the chains' dynamics, the crystallization extent, and would change the nature of the crystallization. At the isothermal temperature of $37^{\circ} \mathrm{C}$, the crystalline structure is melted (as indicated in Figure 5), and the formed temporary shape is fixed by cooling the samples down to $-30^{\circ} \mathrm{C}$. Then, after removing the applied force and reheating the samples, they tend to return to the original shape depending on the extent of crystallization and cross-linking.

Figure 4 and Table 1 show the DMA results of the shape memory analysis. In addition, the schematic image in this figure depicts three different stages of the shape recovery process for unmodified (um) and modified (mod) PU specimens. The melting temperature of the soft segment $\left(+10\right.$ to $\left.+27^{\circ} \mathrm{C}\right)$ is set as the reversible transition temperature to trigger the recovery process, which is well below the body temperature.

The nature of the deformation in the shape memory performance is mainly entropic and the thermodynamic instability, fixed by crystallization, and the
Table 1. Shape recovery and fixity ratios of the prepared in-situ $\mathrm{PU}$ nanocomposites of $\mathrm{PCL}_{1000}-\mathrm{PEG}_{1000^{-}}$ $\mathrm{PCL}_{1000}$ cross-linked by hydroxyl-functionalized (PCL-g-)MWCNTs.

\begin{tabular}{|l|c|c|l|c|c|}
\hline \multicolumn{1}{|c|}{ Sample } & $\begin{array}{c}\boldsymbol{S R} \\
{[\%]}\end{array}$ & $\boldsymbol{S F}$ & \multicolumn{1}{c|}{ Sample } & $\begin{array}{c}\boldsymbol{S R} \\
{[\%]}\end{array}$ & $\boldsymbol{S F}$ \\
\hline Pure & 78 & 100 & - & - & - \\
\hline um- $-0.25 \%$ & 83 & 89 & mod- $0.25 \%$ & 95 & 93 \\
\hline um- $-.50 \%$ & 86 & 93 & mod- $0.50 \%$ & 97 & 98 \\
\hline um- $-1.0 \%$ & 82 & 90 & mod- $-1.0 \%$ & 92 & 96 \\
\hline
\end{tabular}

change in entropy before and after melting temperature are the driving force to recover the original shape [31]. Equations (1) and (2) are used to calculate shape fixity $(S F)$ and recovery $(S R)$ ratios, respectively. The pure sample possessed the highest value of $S F(100 \%)$ and the lowest value of $S R(78 \%)$. The PUs of bare nanoparticles showed lower values of $S F$ and $S R$ ratios compared to PUs of PCL grafted nanoparticles. The mod- $0.5 \%$ sample showed an $S F$ ratio of $98 \%$, whereas this value for um- $0.5 \%$ was 93\%. This is due to the facts that PCL-grafted PU showed a higher elastic modulus and the increased 
extent of hydrogen bonding between urethane linkages made by mobile hard segments between PCL graft and the block copolymer compared to fixed states of urethane linkages in um- $(x)$ samples. Furthermore, the enhanced crystallization extent of the soft segment ( $\mathrm{PCL}_{1000}-\mathrm{PEG}_{1000}-\mathrm{PCL}_{1000}$ ), thermodynamically and kinetically improved fixation of the induced temporary shape.

Shape recovery is defined as an ability to recover the original shape controlled by elasticity as well as physical and chemical cross-linking of the polymer chains. All of the nanocomposites showed more $S R$ values compared to the pure PU since the presence of MWCNTs improves the shape recovery efficiency through increasing the elastic modulus of the samples. The higher $S R$ values of PUs of mod-MWCNTs (92$97 \%$ ) relative to unmodified samples $(82-86 \%)$ is due to the presence of physical cross-linking, the formation of hard domains, besides chemical cross-linking by the nanoparticles. The ability of the MWCNTs to orient themselves in the direction of applied force leads to relaxation of the applied deformation; consequently, increases the dissipation of the transferred energy leading to reduced $S R$ values of the nanocomposite containing $1 \%$ MWCNTs, compared to um$(0.25$ and $0.50 \%)$ [38]. Figure $4 c$ shows the schematic representation of the shape memory process for the two different PU groups.

\subsection{Shape memory mechanisms}

In order to analyze the parameters affecting the shape fixity and recovery, crystallization, chemical thermosetting, and elasticity of the PUs were studied in detail to have a deeper insight into the mechanisms involved in the shape memory performance.

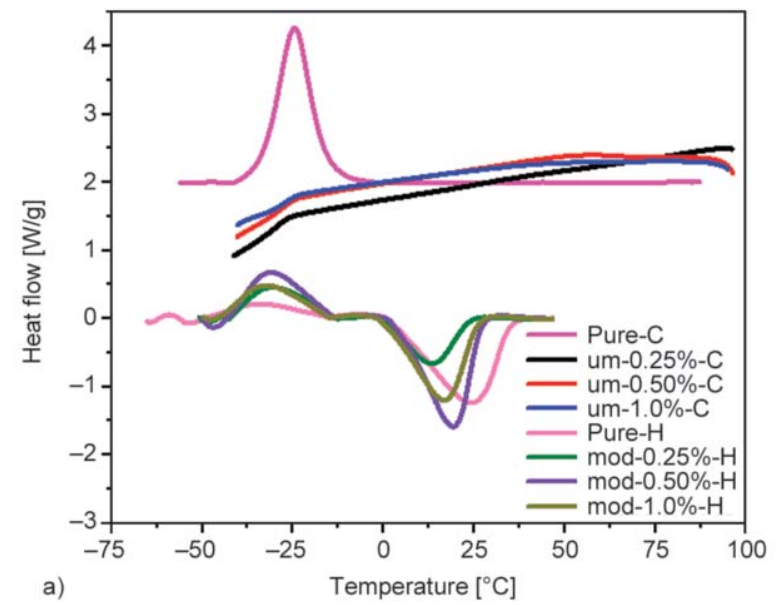

\subsubsection{Crystallization analysis}

Figure 5 show the non-isothermal DSC results. The PU samples of pure and mod-MWCNT( 0.5 and $1 \%)$ showed crystallization in the cooling regime, while polyurethane nanocomposites of unmodified MWCNTs and $0.25 \%$ mod-MWCNT did not show any peak in this regime because of lowered chain dynamic caused by chemical confinement of the polyol [39]. In the second heating regime, the nanocomposites showed cold crystallization after the glass transition temperature. For the PU nanocomposites, the occurrence of crystallization upon heating increases the elastic modulus (an increment in storage modulus after $T_{\mathrm{g}}$ ) by a phenomenon called cold crystallization [27].

The PUs of 0.5 and 1\% PCL-grafted MWCNTs showed a crystallization peak in the cooling regime, and the crystallization process exceeded the second heating regime due to a lack of enough time to complete the crystallization process. The reason for this behavior is the presence of PCL grafts improving the mobility of the soft segments and acting in favor of increasing crystallization kinetics. One of the reasons of the reduction in cold crystallization behavior in these PU nanocomposites is the increase in chain mobility and phase separation, which consequently improved the ability of the soft segment to enter into crystalline regions to form more stable crystallites as seen by increased melting temperature from 10.09 to $24^{\circ} \mathrm{C}$ for PUs of $0.25 \% \mathrm{MWCNT}$ and $0.25 \%$ PCL-g-MWCNT. The characteristic parameters of the DSC thermograms are summarized in Table 2, indicating a gradual change in crystallites stability (increase in $T_{\mathrm{m}}$ ) from unmodified MWCNTs to PCL$g$-MWCNTs.

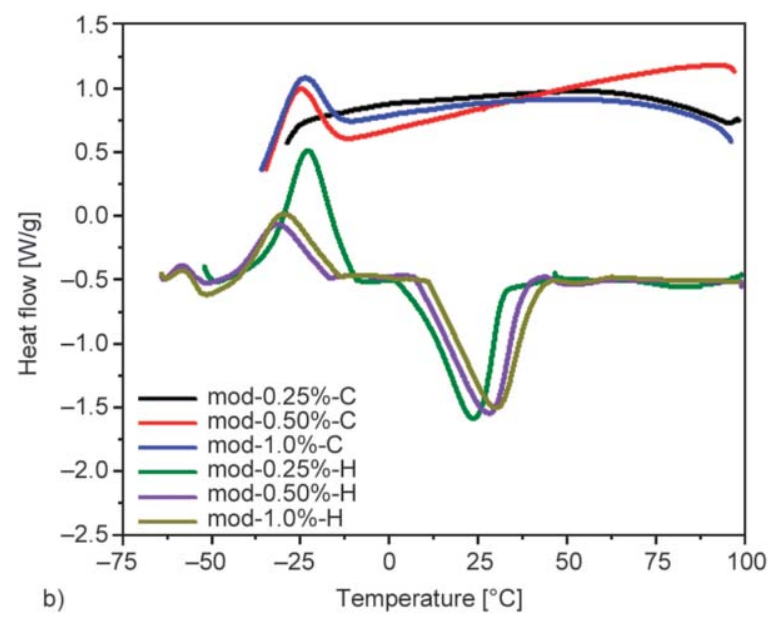

Figure 5. Non-isothermal DSC thermograms of pure and in-situ nanocomposite PUs in cooling (C) and second heating (H) regime for $\mathrm{PCL}_{1000}-\mathrm{PEG}_{1000}-\mathrm{PCL}_{1000}$ soft segment chemically confined between (a) um- and (b) mod-MWCNTs. 
Table 2. DSC characteristics of PU hydrogel samples crosslinked by $\mathrm{OH}$-functionalized MWCNTs (indicated by um for unmodified MWCNT).

\begin{tabular}{|l|c|c|c|c|}
\hline \multicolumn{1}{|c|}{ Sample } & $\begin{array}{c}\boldsymbol{T}_{\mathbf{c}} \\
{\left[{ }^{\circ} \mathbf{C}\right]}\end{array}$ & $\begin{array}{c}\boldsymbol{T}_{\mathbf{c c}} \\
{\left[{ }^{\circ} \mathbf{C}\right]}\end{array}$ & $\begin{array}{c}\boldsymbol{T}_{\mathbf{m}} \\
{\left[{ }^{\circ} \mathbf{C}\right]}\end{array}$ & $\begin{array}{c}\Delta \boldsymbol{H}_{\mathbf{m}} \\
{[\mathbf{J} / \mathbf{g}]}\end{array}$ \\
\hline pure & -23.0 & - & 26.00 & 47.00 \\
\hline um-0.25\% & - & -27.03 & 10.09 & 9.36 \\
\hline um-0.5\% & - & -25.36 & 13.05 & 18.16 \\
\hline um-1\% & - & -23.20 & 15.89 & 21.78 \\
\hline mod-0.25\% & - & -25.00 & 24.00 & 19.64 \\
\hline mod-0.5\% & -27.0 & -27.00 & 26.19 & 28.23 \\
\hline mod-1\% & -25.0 & -28.00 & 28.41 & 38.78 \\
\hline
\end{tabular}

\subsubsection{Isothermal crystallization}

Avrami equation was used to analyze the kinetics of the crystallization of the block copolymer soft segment. The Avrami model considers the relative crystallization $X(t)$ development as a function crystallization time (Equations (7) and (8)):

$$
\begin{aligned}
& X(t)=\frac{Q_{\mathrm{t}}}{Q_{\infty}}=\frac{\int_{0}^{\mathrm{t}} \frac{\mathrm{d} H}{\mathrm{~d} t} \mathrm{~d} t}{\int_{0}^{\infty} \frac{\mathrm{d} H}{\mathrm{~d} t} \mathrm{~d} t} \\
& 1-X(t)=\exp \left(-K t^{\mathrm{n}}\right)
\end{aligned}
$$

where $n$ is the Avrami exponent, and $K$ is a constant parameter indicating the overall kinetic rate of crystallization. Equation (9) represents the linearized form of the model, which is used for double logarithmic plots to fit the isothermal crystallization data. Plots of $\log \left[-\ln \left(1-X_{\mathrm{t}}\right)\right]$ versus $\log t$ result in a linear relation with the line slope of $n$ and intercept of $k$ that can be calculated by fitting the curve to a linear line:

$$
\log [-\ln (1-X(t))]=n \cdot \log t+\log K
$$

Crystallization half-time $\left(t_{0.5}\right.$, considered as the time at which $50 \%$ of the overall crystallization process proceeds) calculated from Equation (10):

$t_{0.5}=\left(\frac{\ln 2}{k}\right)^{1 / \mathrm{n}}$

An Avrami $n$ exponent is a function of the morphology of the growing crystal and the time-dependency of nucleation [40]. An exponent of 3 is related to a spherical structure with an instantaneous nucleation process, and an exponent $n$ between 2 and 3 is attributed to truncated spheres with instantaneous crystal nucleation, with a diffusion-controlled growth. Any variation from the integer numbers of 1,2 , or 3 ,

\begin{tabular}{|c|c|c|c|c|}
\hline & $\begin{array}{c}T_{\mathbf{c}} \\
{\left[{ }^{\circ} \mathrm{C}\right]}\end{array}$ & $n$ & $\begin{array}{c}k \\
{\left[\mathrm{~min}^{-\mathrm{n}}\right]}\end{array}$ & $\begin{array}{c}t_{0.5} \\
{[\mathrm{~min}]}\end{array}$ \\
\hline \multirow{3}{*}{ Pure } & -35 & 2.25 & $7.94 \cdot 10^{-4}$ & 4.31 \\
\hline & -25 & 2.67 & $7.38 \cdot 10^{-3}$ & 2.41 \\
\hline & -15 & 2.37 & $1.58 \cdot 10^{-3}$ & 2.94 \\
\hline \multirow{3}{*}{ um- $0.25 \%$} & -35 & 1.26 & $1.99 \cdot 10^{-4}$ & 11.73 \\
\hline & -25 & 1.43 & $7.94 \cdot 10^{-4}$ & 6.16 \\
\hline & -15 & 1.39 & $5.01 \cdot 10^{-4}$ & 9.53 \\
\hline \multirow{3}{*}{ um- $-0.5 \%$} & -35 & 1.97 & $2.37 \cdot 10^{-4}$ & 11.06 \\
\hline & -25 & 1.78 & $7.62 \cdot 10^{-3}$ & 6.05 \\
\hline & -15 & 2.06 & $9.15 \cdot 10^{-3}$ & 7.84 \\
\hline \multirow{3}{*}{ um-1\% } & -35 & 2.39 & $2.51 \cdot 10^{-4}$ & 10.5 \\
\hline & -25 & 2.05 & $5.58 \cdot 10^{-3}$ & 5.92 \\
\hline & -15 & 2.21 & $1.07 \cdot 10^{-3}$ & 6.96 \\
\hline \multirow{3}{*}{$\bmod -0.25 \%$} & -35 & 2.63 & $8.51 \cdot 10^{-4}$ & 8.32 \\
\hline & -25 & 2.41 & $8.73 \cdot 10^{-3}$ & 5.09 \\
\hline & -15 & 2.34 & $3.98 \cdot 10^{-3}$ & 6.36 \\
\hline \multirow{3}{*}{ mod- $0.5 \%$} & -35 & 2.89 & $6.29 \cdot 10^{-4}$ & 6.47 \\
\hline & -25 & 2.73 & $5.11 \cdot 10^{-3}$ & 4.19 \\
\hline & -15 & 2.86 & $2.89 \cdot 10^{-3}$ & 5.39 \\
\hline \multirow{3}{*}{ mod-1\% } & -35 & 3.17 & $4.76 \cdot 10^{-4}$ & 4.76 \\
\hline & -25 & 3.11 & $1.26 \cdot 10^{-2}$ & 2.67 \\
\hline & -15 & 3.19 & $1.68 \cdot 10^{-3}$ & 3.45 \\
\hline
\end{tabular}

Table 3. Avrami coefficients for isothermal crystallization of the synthesized in-situ PUs.

corresponding to one-, two- or three-dimensional entities that are formed, can be interpreted as the heterogeneous nature of nucleation ( 0 and 1 for spontaneous and completely sporadic nucleation, respectively) [41]. Table 3 shows the Avrami coefficients for isothermal crystallization of the synthesized in-situ PUs at three crystallization temperatures of $-35,-25$, and $-15^{\circ} \mathrm{C}$. The highest kinetic crystal growth constant was for the $T_{\mathrm{c}}$ of $-25^{\circ} \mathrm{C}$ as the highest crystallization rate temperature. Crystallization half-time at each temperature showed a decreasing trend from um- 0.25 to mod- $1.0 \%$, indicating an increased crystallization rate. For the linear (pure) PU sample, the Avrami exponents ( $n$ ) were in the range of 2.25-2.67, indicating a crystal growth with a truncated sphere morphology and instantaneous nucleation. This range indicates a nucleation process, which is diffusion controlled as they are confined between hard domain physical cross-links. The $n$ for in-situ PU nanocomposites of bare MWCNTs was in the range of $1.26-2.39$. To take um- $0.25 \%$ at $-25^{\circ} \mathrm{C}$ as an example, the Avrami exponent of 1.43 indicates a limited crystal growth with heterogeneous nucleation as well as stacking of the lamellas.

For PU nanocomposites of mod-MWCNTs, the Avrami exponent falling within the range of 2.34 to 
3.19 shows a transition from truncated spheres to spherulite crystal morphology growth with a transition from heterogeneous to instantaneous nucleation. The increase in the Avrami exponent for PUs of modified MWCNTs relative to um-MWCNTs is an indication of an increase in the relative rate of thickening growth compared to thickening (i.e., stacking of the lamellas). This is attributed to the increased mobility of PCL segments and the creeping-in mechanism. PCL grafts penetrate into the crystallites through a creeping-in mechanism, resulting in a gradual increase in Avrami exponent as a function of PCL graft content.

\subsubsection{Phase behavior analysis}

In order to analyze the phase behavior of the synthesized polyurethane nanocomposites, to have a better understanding of the crystallization of the soft segments, dynamic mechanical thermal analysis (DMTA) was performed on the samples in the temperature range of -80 to $+100^{\circ} \mathrm{C}$. Figure $6 \mathrm{a}$ represents the
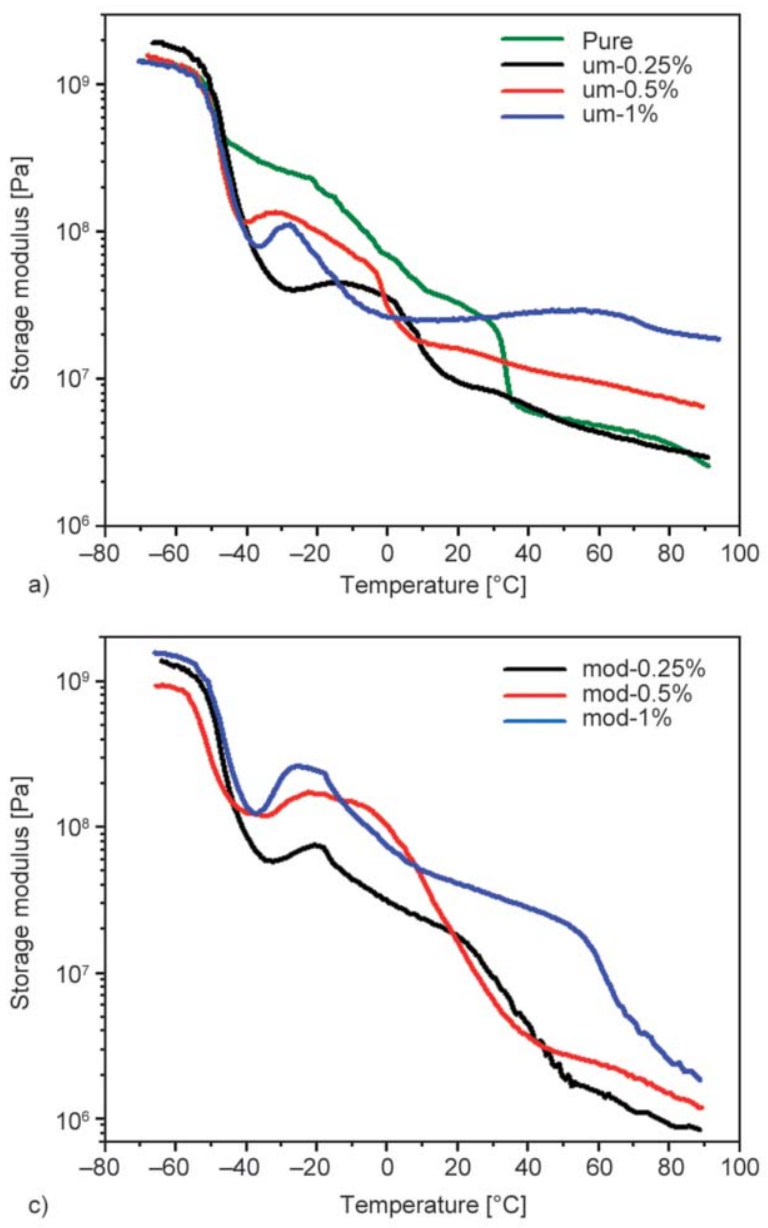

logarithm of storage modulus $v s$. temperature for pure and the PU nanocomposites of $\mathrm{PCL}_{1000^{-}} \mathrm{PEG}_{1000^{-}}$ $\mathrm{PCL}_{1000}$ cross-linked by bare MWCNTs. The PUs showed a reduction in the height of storage modulus compared to the pure sample in the glass transition region, which is attributed to a decrease in the crystallization extent of the nanocomposites [13]. Moreover, the appearance of an increment in the plateau region in the temperature range of -35 and $-15^{\circ} \mathrm{C}$ for the nanocomposites is due to the crystallization of the soft segments in the heating regime (cold crystallization) [27]. The reason for the occurrence of cold crystallization phenomenon is the increased thermal motion of the confined chains after $T_{\mathrm{g}}$ [42]. According to figure $6 \mathrm{c}$, the intensity and temperature range of the increment in the plateau region was increased for PUs of mod-MWCNT.

Regarding Figure 6b, PUs of the um-MWCNTs showed a broad peak in the temperature range of -46 to $-20^{\circ} \mathrm{C}$, while the pure sample with linear structure showed two overlapped peaks as an indication of
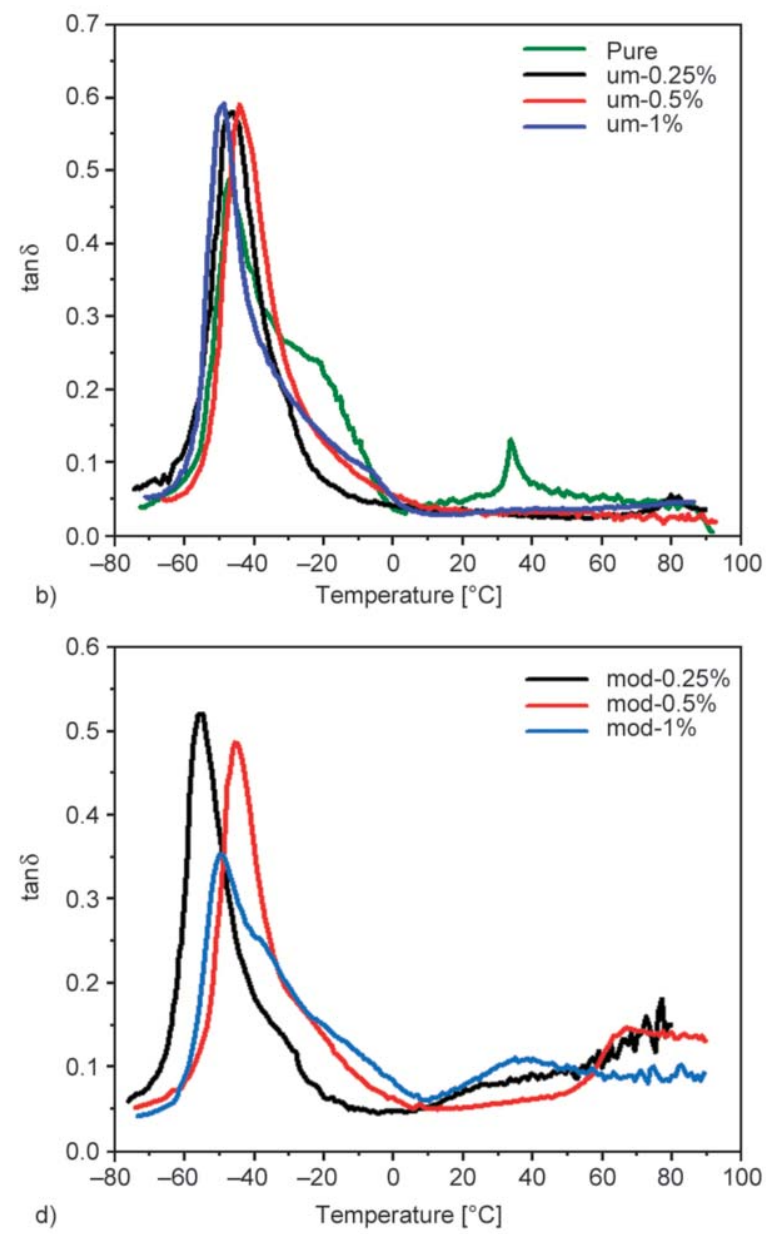

Figure 6. Phase behavior of the prepared hydrogel PU samples; $(a, b)$ storage modulus and tan $\delta$ vs. temperature of PU samples of pure and cross-linked by OH-functionalized MWCNT; $(\mathrm{c}, \mathrm{d})$ storage modulus and $\operatorname{Man} \delta$ vs. temperature of PUs cross-linked by PCL-g-MWCNT, respectively. 
higher phase separation between PCL and PEG blocks. Tan $\delta$ peaks of the PUs of PCL-grafted MWCNTs (Figure 6d) showed a more complex behavior due to the increased freedom of movement for the soft domain chains. The structural changes affected freedom of movement of the soft segments to further intensify the phase separation of $\mathrm{PEG}_{1000}$ $\mathrm{PCL}_{1000}$ block chains.

Compared to PUs of unmodified MWCNTs, nanocomposites of PCL-grafted MWCNTs showed lower soft segment glass transition temperature $\left(T_{\mathrm{g}, \mathrm{ss}}\right)$. $\operatorname{Tan} \delta$ height of the PUs showed a decreasing trend by increasing PCL grafts' content because of increased crystallization upon increasing phase separation of PEG and PCL blocks. Table 4 summarizes the $T_{\mathrm{g}}$ of the soft segment $\left(T_{\mathrm{g}, \mathrm{ss}}\right)$ and $\tan \delta$ peak height of the chemically confined block copolymer.

According to Figure $6 \mathrm{~b}$ and $6 \mathrm{~d}$, in the temperature range of +40 to $+90^{\circ} \mathrm{C}$, PUs of pure and modMWCNTs show a transition, specific of long-range mobility for hard segments $\left(T_{\mathrm{g}, \mathrm{hs}}\right)$. While the PUs of um-MWCNTs did not show any transition, which is due to the chemical attachment of hard segments to the nanoparticles, limiting their mobility and leading to lack of a $T_{\mathrm{g}}$, hs transition.

The presence of PCL grafts intensified the microphase separation of PEG and PCL segments. The phase separation mechanisms of the soft segments for PUs of um- and mod-MWCNTs can be described as a transition from binodal (short-range) to spinodal (long-range) decomposition. Spinodal decomposition produces fluctuations leading to induced chain segmental alignment, which is further intensified by crystallization-assisted phase separation $[42,43]$. The change in phase separation mechanisms of the soft segments is responsible for the morphological change of the crystallites and the rate of crystallization in the PU samples [44]. The increased crystallization

Table 4. $T_{\mathrm{g}, \mathrm{ss}}$ and $\tan \delta$ peak height of PU hydrogel nanocomposites of $\mathrm{PCL}_{1000}-\mathrm{PEG}_{1000}-\mathrm{PCL}_{1000}$ cross-linked by (PCL- $g$-)MWCNTs.

\begin{tabular}{|l|c|c|}
\hline \multicolumn{1}{|c|}{ Sample } & $\begin{array}{c}\boldsymbol{T}_{\mathrm{g}} \\
{[\mathbf{s}]}\end{array}$ & tan $\boldsymbol{\delta}$ peak height \\
\hline Pure & $-47.6 /-27.2$ & $0.50 / 0.39$ \\
\hline um- $0.25 \%$ & -44.1 & 0.61 \\
\hline um- $0.5 \%$ & -45.0 & 0.58 \\
\hline um-1\% & -45.8 & 0.56 \\
\hline mod-0.25\% & -44.8 & 0.52 \\
\hline mod-0.5\% & $-47.8 /-44.9$ & $0.44 / 0.48$ \\
\hline mod-1\% & $-49.2 /-37.5$ & $0.35 / 0.25$ \\
\hline
\end{tabular}

extent and morphological evolution of the crystallites in PUs of mod-MWCNTs to a more stable structure is the reason for higher shape fixity in these samples relative to um-MWCNTs.

\subsection{Cytotoxicity analysis and hMSC culture}

Table 5 presents cell viability analysis by HFF cells, and Figure 7 shows SEM images of cultured hMSCs on the prepared thin films of the $\mathrm{PCL}_{1000^{-}} \mathrm{PEG}_{1000^{-}}$ $\mathrm{PCL}_{1000}$ based PUs. Based on MTT results performed by HFF cells, all the prepared samples proved to have high cell compatibility with a decreasing trend in cell viability by increasing MWCNTs' content from 0 to $1.0 \mathrm{wt} \%$, while PUs of mod-MWCNTs showed lower cell compatibility. The reason for relatively high cytocompatibility can be the presence of a covalent bond between MWCNTs and polyols, prohibiting the migration of the nanoparticles to the culture media. By increasing MWCNTs' content, the probability of migration of MWCNTs increases, causing a reduction in cell viability. The reason for the lower cell viability of PU samples of mod-MWCNTs can be the presence of unreacted MWCNTs due to its lower reaction efficiency relative to bare MWCNTs (as observed during the synthesis of the PU nanocomposites).

Figure 7 show SEM images of the cultured hMSCs onto thin films of the prepared PU nanocomposites of um- and mod-MWCNTs ( 0.25 and $0.50 \%)$. The images indicate that upon increasing MWCNTs' and PCL-graft's content causing a decrease in hydrophilicity of the PUs, adhesion of hMSCs increase, which is in accordance with the relation between hydrophilicity and cell adhesion $[45,46]$.

\section{Conclusions}

PCL-grafted MWCNTs and $\mathrm{PCL}_{1000}-\mathrm{PEG}_{1000}-\mathrm{PCL}_{1000}$ block copolymer were synthesized by ring-opening polymerization of CL monomers in the presence of $\mathrm{Sn}(\mathrm{Oct})_{2}$ as the catalyst. Two groups of reactive in-situ PUs were synthesized based on the tri-block copolymer, chemically cross-linked by hydroxyl

Table 5. The results of the relative cell viability [\%] analysis performed by HFF cells after 24 and $48 \mathrm{hrs}$.

\begin{tabular}{|l|c|c|l|c|c|}
\hline \multicolumn{1}{|c|}{ Sample } & $\mathbf{2 4} \mathbf{~ h r s}$ & $\mathbf{4 8} \mathbf{~ h r s}$ & \multicolumn{1}{c|}{ Sample } & $\mathbf{2 4}$ hrs & $\mathbf{4 8}$ hrs \\
\hline Control & 100 & 100 & Control & 100 & 100 \\
\hline Pure & 92 & 88 & - & - & - \\
\hline um- $0.25 \%$ & 89 & 85 & $\bmod -0.25 \%$ & 87 & 83 \\
\hline um- $0.50 \%$ & 83 & 79 & mod- $0.50 \%$ & 82 & 77 \\
\hline um- $1.0 \%$ & 76 & 69 & $\bmod -1.0 \%$ & 79 & 73 \\
\hline
\end{tabular}




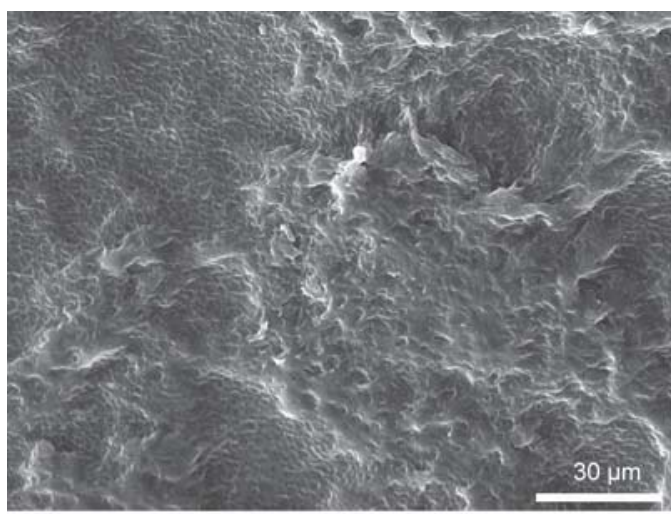

a)

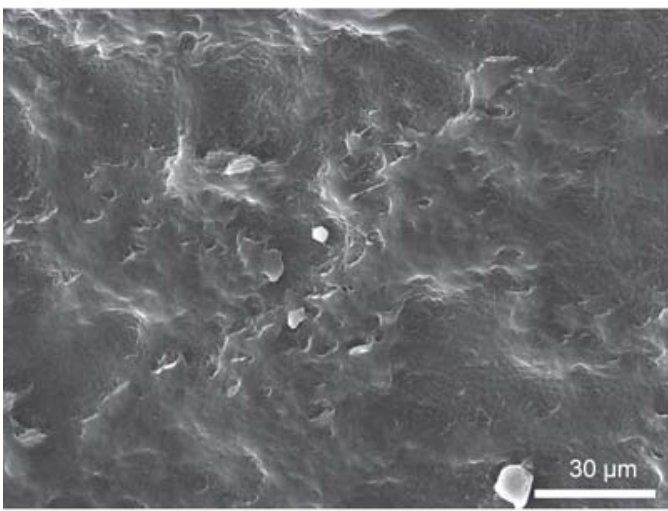

c)

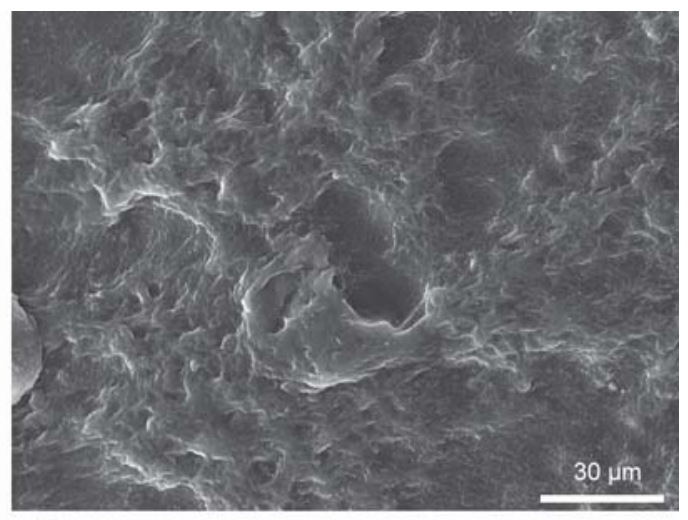

b)

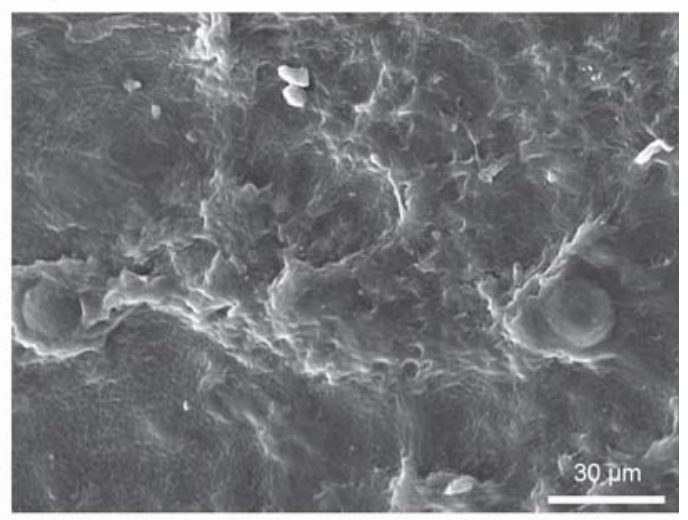

d)

Figure 7. SEM images of cultured hMSCs on $2 \mathrm{D}$ thin films of 0.25 and $0.5 \%$ um- and mod-MWCNT based PUs after 7 days (with a scale bar of $30 \mu \mathrm{m}$ ). a) $0.25 \%$ um b) $0.50 \% \mathrm{um}$, c) $0.25 \% \mathrm{mod}$, d) $0.50 \% \bmod$.

functionalized MWCNTs (um-MWCNT) and PCLgrafted MWCNTs (mod-MWCNT). PCL graft was used to tune the crystallization mechanisms and kinetics of the soft segment to optimize the shape memory performance of the PUs. The presence of PCL grafts changed the thermodynamics and kinetics of crystallization, leading to a wide range of shape fixity (84-100\%) and shape recovery (78$97 \%$ ) values. Besides these changes, using the grafts increased $T_{\mathrm{m}}$, indicating the higher thermal stability of the formed crystallites. Moreover, according to the results of the isothermal DSC measurements, the Avrami exponent ( $n$ ) increased from 1.43 to 3.11, and crystallization half time $\left(t_{0.5}\right)$ showed a decrease from 6.16 to 2.67 min by increasing PCL graft content. Besides, cell viability evaluated by HFF cells demonstrated a proper cytocompatibility for the prepared PU samples. Culturing hMSCs revealed an excellent adhesion and cell spreading, as a function of hydrophilicity.

\section{Acknowledgements}

This research was supported by Amirkabir University of Technology and Pasteur Institute of Iran and the authors do not have any conflict of interest.
Data Availability. The data that support the findings of this study are available from the corresponding author upon reasonable request.

\section{References}

[1] Wang X., Jian W., Lu H., Lau D., Fu Y-Q.: Modeling strategy for enhanced recovery strength and a tailorable shape transition behavior in shape memory copolymers. Macromolecules, 52, 6045-6054 (2019).

https://doi.org/10.1021/acs.macromol.9b00992

[2] Lu H., Huang W. M., Leng J.: On the origin of Gaussian network theory in the thermo/chemo-responsive shape memory effect of amorphous polymers undergoing photo-elastic transition. Smart Materials and Structures, 25, 065004/1-065004/10 (2016).

https://doi.org/10.1088/0964-1726/25/6/065004

[3] Sun L., Huang W. M.: Thermo/moisture responsive shape-memory polymer for possible surgery/operation inside living cells in future. Materials and Design (1980 2015), 31, 2684-2689 (2010).

https://doi.org/10.1016/j.matdes.2009.11.036

[4] Sun L., Huang W. M., Lu H., Lim K. J., Zhou Y., Wang T. X., Gao X. Y.: Heating-responsive shape-memory effect in thermoplastic polyurethanes with low melt-flow index. Macromolecular Chemistry and Physics, 215, 2430-2436 (2014).

https://doi.org/10.1002/macp.201400429 
[5] Jin B., Song H., Jiang R., Song J., Zhao Q., Xie T.: Programming a crystalline shape memory polymer network with thermo- and photo-reversible bonds toward a single-component soft robot. Science Advances, 4, eaao3865/1-eaao3865/7 (2018).

https://doi.org/10.1126/sciadv.aao3865

[6] Kim B. K., Shin Y. J., Cho S. M., Jeong H. M.: Shapememory behavior of segmented polyurethanes with an amorphous reversible phase: The effect of block length and content. Journal of Polymer Science Part B: Polymer Physics, 38, 2652-2657 (2000).

https://doi.org/10.1002/10990488(20001015)38:20<2652::AID-POLB50>3.0.CO;2-3

[7] Lendlein A., Kelch S.: Shape-memory polymers. Angewandte Chemie International Edition, 41, 2034-2057 (2002).

https://doi.org/10.1002/1521-

3773(20020617)41:12<2034::AID-ANIE2034>3.0.CO;2-M

[8] Hood M. A., Wang B., Sands J. M., La Scala J. J., Beyer F. L., Li C. Y.: Morphology control of segmented polyurethanes by crystallization of hard and soft segments. Polymer, 51, 2191-2198 (2010).

https://doi.org/10.1016/j.polymer.2010.03.027

[9] Wang X., Hu Y., Song L., Yang H., Xing W., Lu H.: In situ polymerization of graphenenanosheets and polyurethane with enhanced mechanical and thermal properties. Journal of materials Chemistry, 21, 4222-4227 (2011).

https://doi.org/10.1039/C0JM03710A

[10] Panahi-Sarmad M., Goodarzi V., Amirkiai A., Noroozi M., Abrisham M., Dehghan P., Shakeri Y., KarimpourMotlagh N., Hajipoor F. P., Khonakdar H. A., Asefnejad A.: Programing polyurethane with systematic presence of graphene-oxide (GO) and reduced graphene-oxide (rGO) platelets for adjusting of heat-actuated shape memory properties. European Polymer Journal, 118, 619-632 (2019).

https://doi.org/10.1016/j.eurpolymj.2019.06.034

[11] Ghoshal T., Biswas S., O’Regan C., Holmes J. D., Morris M. A.: Nanophase separation and structural evolution of block copolymer films: A 'green' and 'clean' supercritical fluid approach. Nano Research, 8, 1279-1292 (2015).

https://doi.org/10.1007/s12274-014-0616-7

[12] Erukhimovich I.: The latest development of the weak segregation theory of microphase separation in block copolymers. in 'Nanostructured soft matter' (ed.: Zvelindovsky A. V.) Springer, Dordrecht, 327-370 (2007). https://doi.org/10.1007/978-1-4020-6330-5 11

[13] Sahebi Jouibari I., Haddadi-Asl V., Mirhosseini M. M.: Effect of nanofiller content and confined crystallization on the microphase separation kinetics of polyurethane nanocomposites. Polymer Composites, 40, E422-E430 (2019).

https://doi.org/10.1002/pc.24717
[14] Kim J-S., Lee Y. J., Coote J. P., Stein G. E., Kim B. J.: Confined, templated, and break-through crystallization modes in poly(3-dodecylthiophene)-block-poly(ethyl methacrylate) block copolymers. Macromolecules, 52, 4475-4482 (2019).

https://doi.org/10.1021/acs.macromol.9b00816

[15] Pei A., Malho J-M., Ruokolainen J., Zhou Q., Berglund L. A.: Strong nanocomposite reinforcement effects in polyurethane elastomer with low volume fraction of cellulose nanocrystals. Macromolecules, 44, 4422-4427 (2011). https://doi.org/10.1021/ma200318k

[16] Liu Y., Li Y., Yang G., Zheng X., Zhou S.: Multi-stimulus-responsive shape-memory polymer nanocomposite network cross-linked by cellulose nanocrystals. ACS Applied Materials and Interfaces, 7, 4118-4126 (2015). https://doi.org/10.1021/am5081056

[17] Fu N., Liao J., Lin S., Sun K., Tian T., Zhu B., Lin Y.: PCL-PEG-PCL film promotes cartilage regeneration in vivo. Cell Proliferation, 49, 729-739 (2016). https://doi.org/10.1111/cpr.12295

[18] Shahrousvand M., Sadeghi G. M. M., Salimi A., Nourany M.: Bulk synthesis of monodisperse and highly biocompatible poly( $\varepsilon$-caprolactone)-diol by transesterification side-reactions. Polymer-Plastics Technology and Engineering, 57, 492-499 (2018). https://doi.org/10.1080/03602559.2016.1211690

[19] Li G., Li D., Niu Y., He T., Chen K. C., Xu K.: Alternating block polyurethanes based on PCL and PEG as potential nerve regeneration materials. Journal of Biomedical Materials Research Part A, 102, 685-697 (2014). https://doi.org/10.1002/jbm.a.34732

[20] Torabinejad B., Mohammadi-Rovshandeh J., Davachi S. M., Zamanian A.: Synthesis and characterization of nanocomposite scaffolds based on triblock copolymer of L-lactide, $\varepsilon$-caprolactone and nano-hydroxyapatite for bone tissue engineering. Materials Science and Engineering: C, 42, 199-210 (2014). https://doi.org/10.1016/j.msec.2014.05.003

[21] Dobrzynski P., Li S., Kasperczyk J., Bero M., Gasc F., Vert M.: Structure-property relationships of copolymers obtained by ring-opening polymerization of glycolide and $\varepsilon$-caprolactone. Part 1 . Synthesis and characterization. Biomacromolecules, 6, 483-488 (2005). https://doi.org/10.1021/bm0494592

[22] Kowalski A., Libiszowski J., Biela T., Cypryk M., Duda A., Penczek S.: Kinetics and mechanism of cyclic esters polymerization initiated with tin(II) octoate. Polymerization of $\varepsilon$-caprolactone and L,L-lactide $c o$-initiated with primary amines. Macromolecules, 38, 8170-8176 (2005). https://doi.org/10.1021/ma050752j

[23] Divakaran A. V., Torris A. T. A., Lele A. K., Badiger M. V.: Porous poly(ethylene glycol)-polyurethane hydrogels as potential biomaterials. Polymer International, 64, 397-404 (2015).

https://doi.org/10.1002/pi.4802 
[24] Shokrolahi F., Yeganeh H.: Soft segment composition and its influence on phase-separated morphology of PCL/PEG-based poly(urethane urea)s. Iranian Polymer Journal, 23, 505-512 (2014).

https://doi.org/10.1007/s13726-014-0245-8

[25] Liu C. B., Gong C. Y., Huang M. J., Wang J. W., Pan Y. F., Zhang Y. D., Li G. Z., Gou M. L., Wang K., Tu M. J., Wei Y. Q., Qian Z. Y.: Thermoreversible gel-sol behavior of biodegradable PCL-PEG-PCL triblock copolymer in aqueous solutions. Journal of Biomedical Materials Research Part B: Applied Biomaterials, 84, 165 175 (2008).

https://doi.org/10.1002/jbm.b.30858

[26] Peponi L., Navarro-Baena I., Sonseca A., Gimenez E., Marcos-Fernandez A., Kenny J. M.: Synthesis and characterization of PCL-PLLA polyurethane with shape memory behavior. European Polymer Journal, 49, 893 903 (2013).

https://doi.org/10.1016/j.eurpolymj.2012.11.001

[27] Zheng Z., Cui Z., Si J., Yu S., Wang Q., Chen W., Turng L-S.: Modification of 3-D porous hydroxyapatite/thermoplastic polyurethane composite scaffolds for reinforcing interfacial adhesion by polydopamine surface coating. ACS Omega, 4, 6382-6391 (2019).

https://doi.org/10.1021/acsomega.9b00404

[28] Hesami M., Jalali-Arani A.: Cold crystallization behavior of poly(lactic acid) in its blend with acrylic rubber; The effect of acrylic rubber content. Polymer International, 66, 1564-1571 (2017).

https://doi.org/10.1002/pi.5414

[29] Amici G., Caraglio M., Orlandini E., Micheletti C.: Topologically linked chains in confinement. ACS Macro Letters, 8, 442-446 (2019).

https://doi.org/10.1021/acsmacrolett.9b00114

[30] Phua S. L., Yang L., Toh C. L., Huang S., Tsakadze Z., Lau S. K., Mai Y-W., Lu X.: Reinforcement of polyether polyurethane with dopamine-modified clay: The role of interfacial hydrogen bonding. ACS Applied Materials and Interfaces, 4, 4571-4578 (2012).

https://doi.org/10.1021/am300947b

[31] Jafari S., Nourany M., Zakizadeh M., Taghilou A., Ranjbar H. A., Noormohammadi F.: The effect of controlled phase separation of PEG/PCL-2000 homopolymer polyols using their $\mathrm{PCL}_{500}-\mathrm{PEG}_{1000}-\mathrm{PCL}_{500}$ triblock copolymer and CNCs in the final polyurethane hydrogels on their shape memory behavior. Composites Communications, 9, 194-202 (2020).

https://doi.org/10.1016/j.coco.2020.03.016

[32] Kricheldorf H. R., Rost S.: Biodegradable multiblock copolyesters prepared from $\varepsilon$-caprolactone, L-lactide, and trimethylene carbonate by means of bismuth hexanoate. Macromolecules, 38, 8220-8226 (2005).

https://doi.org/10.1021/ma050439h

[33] Zhou S., Deng X., Yang H.: Biodegradable poly(ع-caprolactone)-poly(ethylene glycol) block copolymers: Characterization and their use as drug carriers for a controlled delivery system. Biomaterials, 24, 3563-3570 (2003). https://doi.org/10.1016/S0142-9612(03)00207-2
[34] Feng H., Dong C-M.: Preparation and characterization of chitosan-graft-poly( $\varepsilon$-caprolactone) with an organic catalyst. Journal of Polymer Science Part A: Polymer Chemistry, 44, 5353-5361 (2006).

https://doi.org/10.1002/pola.21625

[35] Li Y., Chen H., Liu D., Wang W., Liu Y., Zhou S.: pHresponsive shape memory poly(ethylene glycol)-poly ( $\varepsilon$-caprolactone)-based polyurethane/cellulose nanocrystals nanocomposite. ACS Applied Materials and Interfaces, 7, 12988-12999 (2015).

https://doi.org/10.1021/acsami.5b02940

[36] Sun Y-C., Cai S., Ren J., Naguib H. E.: Room temperature deformable shape memory composite with finetuned crystallization induced via nanoclay particles. Journal of Polymer Science Part B: Polymer Physics, 55, 1197-1206 (2017). https://doi.org/10.1002/polb.24370

[37] Xia L., Wu H., Qiu G.: Shape memory behavior of carbon nanotube-reinforced trans-1,4-polyisoprene and lowdensity polyethylene composites. Polymers for Advanced Technologies, 31, 107-113 (2020).

https://doi.org/10.1002/pat.4751

[38] Gu S., Yan B., Liu L., Ren J.: Carbon nanotube-polyurethane shape memory nanocomposites with low trigger temperature. European Polymer Journal, 49, 38673877 (2013).

https://doi.org/10.1016/j.eurpolymj.2013.10.007

[39] Jouibari I. S., Haddadi-Asl V., Mirhosseini M. M.: A novel investigation on micro-phase separation of thermoplastic polyurethanes: Simulation, theoretical, and experimental approaches. Iranian Polymer Journal, 28, 237-250 (2019). https://doi.org/10.1007/s13726-019-00695-6

[40] Sanandaji N., Bretzler V., Schmelter S., Olsson R. T., Hedenqvist M. S., Gedde U. W.: Confined space crystallisation of poly( $\varepsilon$-caprolactone) in controlled pore glasses. European polymer journal, 49, 2073-2081 (2013). https://doi.org/10.1016/j.eurpolymj.2013.04.034

[41] Tol R. T., Mathot V. B. F., Reynaers H., Goderis B., Groeninckx G.: Confined crystallization phenomena in immiscible polymer blends with dispersed micro-and nanometer sized PA6 droplets, Part 4: Polymorphous structure and (meta)-stability of PA6 crystals formed in different temperature regions. Polymer, 46, 2966-2977 (2005).

https://doi.org/10.1016/j.polymer.2005.02.021

[42] Ranjbar H. A., Nourany M., Mollavali M., Noormohammadi F., Jafari S.: Stimuli-responsive polyurethane bionanocomposites of poly(ethylene glycol)/ poly( $\varepsilon$-caprolactone) and [poly( $\varepsilon$-caprolactone)-grafted-]cellulose nanocrystals. Polymers for Advanced Technologies, in press (2020).

https://doi.org/10.1002/pat.5062 
[43] Zhang X., Wang Z., Muthukumar M., Han C. C.: Fluctuation-assisted crystallization: In a simultaneous phase separation and crystallization polyolefin blend system. Macromolecular Rapid Communications, 26, 12851288 (2005)

https://doi.org/10.1002/marc.200500304

[44] Niu Y., Yang L., Wang H., Wang Z.: Criteria of process optimization in binary polymer blends with both phase separation and crystallization. Macromolecules, 42, 7623-7626 (2009).

https://doi.org/10.1021/ma901543c
[45] Wei J., Igarashi T., Okumori N., Igarashi T., Maetani T., Liu B., Yoshinari M.: Influence of surface wettability on competitive protein adsorption and initial attachment of osteoblasts. Biomedical Materials, 4, 045002/1045002/8 (2009).

https://doi.org/10.1088/1748-6041/4/4/045002

[46] Ferrari M., Cirisano F., Morán M. C.: Mammalian cell behavior on hydrophobic substrates: Influence of surface properties. Colloids and Interfaces, 3, 3020048/13020048/16 (2019).

https://doi.org/10.3390/colloids3020048 